\title{
LA VIOLENCIA EN LAS ESCUELAS
}

\author{
Edith Moreno Artal \\ Maestra del Instituto Educativo Moderno
}

Recibido 30-VIII-2004 • Aceptado 7-IX-2004

\begin{abstract}
Resumen: Las instituciones educativas deben enfrentar un nuevo reto: el manejo de la violencia en sus instalaciones. La violencia es un problema que tiene muchas causas tanto sociales como psicológicas, las cuales son esbozadas en este artículo a la luz de los postulados de Martín-Baró y Bandura, premisas que son debidamente contextualizadas en la realidad costarricense.

A continuación se ofrecen alternativas para enfrentar el problema de la violencia, basadas en las experiencias realizadas en otros países y en las acciones propuestas por el Ministerio de Educación Pública.

El problema de la violencia, por su complejidad, requiere un trabajo interdisciplinario en las instituciones, además, precisa involucrar a la comunidad y las familias y gestionar acciones de otras instancias gubernamentales orientadas a disminuir problemas sociales como el hacinamiento y la pobreza en que viven algunas familias.
\end{abstract}

Palabras clave: Educación, violencia, educación para la paz.

\begin{abstract}
Schools must face a new challenge: the management of violence. Violence is a problem that has both social and psychological causes. This article outlines such causes in the light of the ideas of Martín-Baró and Bandura on this topic. These causes are properly fit into Costa Rican reality. Different alternatives to face the problem of violence in schools based on (1) experiences in other countries and (2) the course of action proposed by the Costa Rican Ministry of Education are offered. Because of its complexity, this problem requires an interdisciplinary effort from schools, community and families. The government should also encourage action to reduce social problems such as poverty and inadequate housing which affect many students' families.
\end{abstract}

Key words: Education, violence, education for peace.

\section{Introducción}

Las acciones violentas dentro de las instituciones educativas son una realidad que preocupa a docentes, padres y madres de familia y, en general, a toda la sociedad. Dicha realidad se ve reflejada en los artículos que regularmente ha estado publicando el periódico "La Nación", en la creación del "Plan Nacional para la prevención y atención de la violencia en y desde el Sistema Educativo" del Ministerio de Educación Pública y, en los textos referidos al tema, publicados en revistas. El tema también ha sido abordado en conferencias e investigaciones que intentan explicar e intervenir en esta situación, tanto en nuestro país, como en Europa, Estados Unidos y América del Sur.

El objetivo de este artículo es ofrecer lineamientos para enfrentar el problema de la violencia en los centros escolares y reflexionar en torno a los factores sociales y psicológicos que influyen en las respuestas agresivas o violentas de los niños y las niñas.

El Ministerio de Educación Pública cuenta con datos estadísticos de la violencia en las escuelas, obtenidos por primera vez a través del cuadro de matrícula final del año 2002, período en el que se contabilizaron 83.800 casos de violencia estudiantil, cuya gran mayoría se reportó en la escuela primaria. El periódico La 
Nación del día 8 de octubre del 2003, en la página 4 , presenta las estadísticas del Ministerio de Educación y la opinión de algunas docentes respecto de este problema. Una de ellas piensa que la violencia obedece a la falta de autoridad que tiene la planta docente debido al reglamento de disciplina, ya que éste limita la posibilidad de aplicar acciones correctivas y hace que, por ejemplo, el dejar a las niñas o a los niños sin recreo sea considerado como una trasgresión a los derechos del estudiantado. También se señala, como causa de la violencia, el desinterés de la familia por el desempeño escolar de sus hijas e hijos, ya que los progenitores no acostumbran asistir a las reuniones a retirar notas, ni cuando son citados por el cuerpo docente.

Por su parte, otra educadora manifestó que las acciones correctivas, como mandar a los niños o las niñas a la casa, no surten el efecto esperado y por ello, prefiere el diálogo conciliador cuando se producen episodios de agresión así como las charlas sobre temas como el autoestima y el respeto a las personas.

Según la estadística del Ministerio de Educación Pública, los episodios agresivos se dan en cualquier lugar donde exista una interrelación de los escolares, tanto dentro como fuera del centro escolar. Asimismo existe una diferencia entre la cantidad de agresiones declaradas en preescolar y en I y II ciclos, ya que en el nivel de preescolar el número de agresiones fuera de la institución es menor, respecto de las agresiones que se producen en el aula y los pasillos. En I y II ciclos, es mayor el número de agresiones que se dan fuera de la escuela, respecto de las que se presentan en los pasillos y en el aula. En preescolar la mayor cantidad de agresiones reportadas se dan en el aula, en cambio, en I y II ciclos de educación primaria, la mayor cantidad de agresiones se da en los pasillos de la institución. En ambas poblaciones existen menos agresiones ocurridas en el comedor, baño y gimnasio (Ministerio de Educación Pública, 2003).
El cuadro 1 presenta un detalle de los datos antes mencionados.

En las estadísticas se señalan diferentes tipos de agresiones o situaciones de violencia, entre ellas, la violencia verbal o escrita, la violencia física, la destrucción de materiales, los robos y otras modalidades de agresión. En los grupos de preescolar y I y II ciclos de primaria, la agresión verbal es la que cuenta con el número mayor de casos reportados, seguida por el número de casos referidos a la agresión física. En primaria, la destrucción de materiales, los robos y la agresión escrita no registran mayor diferencia en cuanto a los casos reportados, aunque es mayor la cifra de robos respecto de las agresiones verbales y escritas. En Preescolar, la cifra de casos relativos a la destrucción de materiales representa casi la mitad de los casos reportados en primaria (Ministerio de Educación Pública, 2003).

El cuadro 2 ilustra con mayor precisión los aspectos antes mencionados.

En definitiva, conceptuar lo que se entiende por violencia puede ayudar a comprender las posturas que se toman al respecto, cuando, por ejemplo, el adulto prefiere no intervenir si lo que se ha dado entre el alumnado es sólo un intercambio de palabras fuertes que no llega a traducirse en agresión física.

En nuestro país, una de las instituciones que trabaja en la prevención de la violencia infantil es la Fundación Paniamor, organización que a partir de la definición de diversos autores acerca del tema, expone su concepto de violencia desde la perspectiva de los derechos humanos de las personas menores de edad. Esta conceptualización es amplia, ya que implica todas aquellas situaciones que lleven al irrespeto de los derechos de la niñez, e incluye la agresión física y psicológica, por ejemplo, omitir acciones que promuevan el desarrollo del potencial de la persona menor de edad (Brenes, R. y Grillo, M. 1998:25).

El diccionario de La Lengua Española Larousse (2003) define la violencia como actuar haciendo uso excesivo de la 


\section{Cuadro $\mathrm{N}^{\circ} 1$}

Lugares donde se dan agresiones o situaciones de violencia entre estudiantes, en instituciones del sistema educativo, por lugar y nivel de enseñanza, 2002

\begin{tabular}{|c|c|c|c|c|c|c|}
\hline & Total & Preescolar & $\begin{array}{l}\text { I y II } \\
\text { Ciclos }\end{array}$ & $\begin{array}{l}\text { III Ciclo y } \\
\text { Educ. } \\
\text { Diversif. }\end{array}$ & $\begin{array}{c}\text { Educación } \\
\text { Especial }\end{array}$ & $\begin{array}{l}\text { Escuelas } \\
\text { Nocturnas }\end{array}$ \\
\hline & & \multicolumn{5}{|c|}{ Cifras absolutas } \\
\hline Fuera de la Institución & 2473 & 434 & 1777 & 253 & 3 & 6 \\
\hline Pasillos & 2325 & 657 & 1397 & 255 & 10 & 6 \\
\hline Aula & 1772 & 689 & 835 & 235 & 8 & 5 \\
\hline Comedor & 457 & 118 & 302 & 32 & 4 & 1 \\
\hline Baño & 291 & 47 & 141 & 99 & 2 & 2 \\
\hline \multirow[t]{2}{*}{ Gimnasio } & 264 & 51 & 151 & 62 & - & - \\
\hline & & \multicolumn{5}{|c|}{ Cifras relativas ${ }^{1 /}$} \\
\hline Fuera de la Institución & 36,2 & 19,0 & 45,5 & 43,6 & 7,5 & 60,0 \\
\hline Pasillos & 34,1 & 28,7 & 35,8 & 44,0 & 25,0 & 60,0 \\
\hline Aula & 26,0 & 30,1 & 21,4 & 405 & 20,0 & 50,0 \\
\hline Comedor & 6,7 & 5,2 & 7,7 & 5,5 & 10,0 & 10,0 \\
\hline Baño & 4,3 & 2,1 & 3,6 & 17,1 & 5,0 & 20,0 \\
\hline Gimnasio & 3,9 & 2,2 & 3,9 & 10,7 & - & - \\
\hline
\end{tabular}

1/ Cifras calculadas respecto al número de instituciones de cada Nivel de Enseñanza.

Fuente: Ministerio de Educación Pública, 2002.

\section{Cuadro $\mathrm{N}^{\circ} 2$}

Tipos de agresiones o situaciones de violencia entre estudiantes, en instituciones del sistema educativo, por tipo y nivel de enseñanza, 2002

\begin{tabular}{|c|c|c|c|c|c|c|}
\hline & Total & Preescolar & $\begin{array}{l}\text { I y II } \\
\text { Ciclos }\end{array}$ & $\begin{array}{c}\text { III Ciclo y } \\
\text { Educ. } \\
\text { Diversif. }\end{array}$ & $\begin{array}{c}\text { Educación } \\
\text { Especial }\end{array}$ & $\begin{array}{l}\text { Escuelas } \\
\text { Nocturnas }\end{array}$ \\
\hline & & \multicolumn{5}{|c|}{ Cifras absolutas } \\
\hline Verbal & 4053 & 1105 & 2509 & 419 & 12 & 7 \\
\hline Física & 2772 & 892 & 1607 & 256 & 11 & 6 \\
\hline Destrucción de Materiales & 880 & 227 & 495 & 151 & 5 & 2 \\
\hline Robos & 869 & 86 & 549 & 230 & 1 & 3 \\
\hline Escrita & 642 & 21 & 421 & 198 & - & 2 \\
\hline \multirow[t]{2}{*}{ Otro } & 83 & 21 & 38 & 21 & 1 & 2 \\
\hline & & \multicolumn{5}{|c|}{ Cifras relativas ${ }^{1 /}$} \\
\hline Verbal & 59,4 & 48,3 & 64,3 & 72,2 & 30,0 & 70,0 \\
\hline Física & 40,6 & 39,0 & 41,2 & 44,1 & 27,5 & 60,0 \\
\hline Escrita & 12,9 & 9,9 & 12,7 & 26,0 & 12,5 & 20,0 \\
\hline Robos & 12,7 & 3,8 & 14,1 & 39,7 & 2,5 & 30,0 \\
\hline Destrucción de Materiales & 9,4 & 0,9 & 10,8 & 34,1 & & 20,0 \\
\hline Otro & 1,2 & 0,9 & 1,0 & 3,6 & 2,5 & 20,0 \\
\hline
\end{tabular}

1/ Cifras calculadas respecto al número de instituciones de cada Nivel de Enseñanza.

Nota: Este cuadro se refiere a los tipos de agresión o situaciones de violencia entre estudiantes que se dan en las diferentes instituciones. Cada institución podía indicar más de un tipo de agresión o situación de violencia.

Fuente: Ministerio de Educación Pública, 2002. 
fuerza física y como la acción injusta con que se ofende o perjudica a alguien. Esta definición es consecuente con el contexto en que estamos reflexionando, ya que no sólo considera el uso de la fuerza física sino que también las acciones injustas que perjudican a los demás. En la convivencia diaria sobran ejemplos del uso de la fuerza por parte de países militarizados, política y económicamente poderosos, sobre otras naciones más débiles. De igual modo, en el terreno de las relaciones interpersonales, es común observar conductas como la típica "serruchada de piso", la cual, inclusive ha llegado a considerarse como parte de nuestra idiosincrasia. El maltrato a las personas de todas las edades se reporta, ampliamente en los medios de comunicación, sobre todo la violencia contra las mujeres que anualmente mueren atacadas por su pareja, tema que por fin ha logrado dejar de ser tabú y ya se perfilan condenas para los agresores.

En América Latina, uno de los teóricos que ha tratado el tema de la violencia a fondo es Ignacio Martín-Baró (1995), quien entiende la violencia como un fenómeno más amplio que la agresión. Dicho autor considera que cualquier acto en que se aplique una dosis excesiva de fuerza es considerado violento, y entiende la agresión como una forma de violencia en la que alguien aplica la fuerza contra otra persona, de manera intencional, para causarle daño. Según su criterio es posible hablar de violencia estructural o institucional, porque las estructuras sociales pueden aplicar una fuerza para hacer que las personas actúen en contra de su propia decisión; pero no es posible hablar de agresión estructural, ya que las estructuras sociales tienen como objetivo beneficiar al grupo social dominante, implicando, de paso, la violencia contra los grupos sociales dominados.

Para tratar de explicar las causas de la violencia escolar y dilucidar las posibles formas de abordar el problema, se ha organizado la información en los siguientes apartados:
- Factores sociológicos y psicológicos de la violencia, apartado que expone los supuestos sociológicos de la violencia según Martín-Baró y los principios de modelamiento y comportamiento agresivo de Bandura.

- Lineamientos para enfrentar el problema de la violencia en los centros escolares. Este apartado se fundamenta en las propuestas desarrolladas en la Universidad de Nueva York y la Universidad de Sevilla, además se sustenta con la reflexión de personal docente entrevistado, el cual ha tenido que enfrentar situaciones de violencia en sus aulas, y en situaciones divulgadas por los diferentes medios de comunicación.

\section{Factores sociológicos y psicológicos de la violencia}

La violencia, como estado de comportamiento producido fuera de las leyes establecidas, afecta directamente el desarrollo emocional y social del ser humano. A continuación, se reflexiona acerca de los factores sociológicos y psicológicos que, de una u otra manera, influyen en el surgimiento de situaciones de violencia en los centros escolares. La reflexión en torno a dichos factores se respalda con la opinión de educadoras y educadores entrevistados para este fin.

\subsection{Factores sociológicos de la violencia}

La interpretación de las palabras violencia y agresión a veces resulta confusa porque si bien es común dar una carga negativa al concepto de violencia, la agresión se entiende, más bien, como una cualidad positiva en el desempeño de las personas de algunas profesiones. El lenguaje cotidiano está teñido por esta concepción y así, es común escuchar que una 
persona profesional, para triunfar en estos días, debe comportarse de manera "agresiva”. Esta percepción otorga una valoración positiva a la agresividad. La organización capitalista de la sociedad valora como negativas las acciones que van en contra del orden establecido y; por lo tanto, sólo esas son consideradas violentas y agresivas; en cambio, valora como aceptable y positiva la agresión efectuada en contra de las personas que desean cambiar el ordenamiento establecido. Martín-Baró lo expresa claramente: "Así, la dignificación de la agresividad como característica deseable en el mundo de los negocios pudiera expresar el intento por justificar lo que son prácticas que, al desnudo, resultan socialmente inadmisibles". (Martín-Baró. 1995, 368). En este contexto, la práctica de actitudes solidarias con las compañeras y los compañeros de trabajo, por ejemplo, señalarles algunas faltas que han cometido o darles consejos para propiciar su superación, parecen quedar por fuera, ya que es más efectivo descalificarlos o descalificarlas ante los superiores para conseguir puntos a favor. Al interpretar la agresividad como la acción que daña con intención, en el mundo de los negocios, la deslealtad llega a percibirse como una cualidad y no como una conducta impregnada por el valor negativo de la agresión.

La violencia puede analizarse desde diferentes puntos de vista, y para este efecto se tomarán tres presupuestos señalados por Martín-Baró, (1995), los cuales ayudan a entender que la violencia tiene muchas aristas, e involucra creencias, actitudes y por supuesto, las estructuras sociales.

El primer presupuesto, plantea que la violencia presenta muchísimas formas y diferencias significativas entre cada una de ellas, las cuales deben tenerse en cuenta a la hora de analizar una acción violenta. Estas diferencias son las siguientes:

1. La violencia estructural dada por todo ordenamiento social es diferente a la violencia interpersonal, que puede ser expresión de la violencia estructural o tener un carácter autónomo.

2. La violencia educativa por la que los adultos obligan a los niños $\mathrm{y}$ las niñas a realizar actividades o ejercicios es diferente a la violencia personal cuando es la misma persona la que se obliga para sobreponer su repugnancia ante alguna tarea, para poder superarse.

3. La agresión institucional por medio de la cual un ejército ataca a una población civil, será diferente a la agresión interpersonal producto de la rabia con la otra persona.

4. La agresión física que tiende a matar o herir a otra persona, es diferente de la agresión moral, simbólica, el insulto o la calumnia por la que se trata de desprestigiar a la persona.

Al entrevistar a algunos maestros y maestras de primaria y preescolar acerca de estos presupuestos, manifestaron que a veces el ordenamiento del sistema escolar resulta violento pues obliga a la población estudiantil a obtener un mismo rendimiento académico, sin tener en cuenta las diferencias contextuales de cada estudiante y sus diferentes estilos de aprendizaje. Algunos docentes opinaron que, en ocasiones, los padres y las madres de familia proceden con violencia, al exigir a sus hijos permanecer en una determinada institución educativa, por el prestigio que esto les confiere, a pesar de que sus hijos e hijas no tienen las cualidades para permanecer en ella. Este tipo de imposiciones implica sufrimiento para el niño o la niña, puesto que no puede rendir como se espera. Cabe destacar que algunas madres y algunos padres de familia se oponen a que sus hijos o hijas tengan adecuaciones curriculares, y se niegan a aceptar las limitaciones que muestran sus hijos e hijas en el desempeño de algunas actividades académicas. Respecto de la actuación agresiva de los policías algunas personas entrevistadas indicaron que ese 
tipo de violencia es válida en tanto permite garantizar la seguridad de la mayor parte de la población; por el contrario otras de las personas entrevistadas manifestaron que al tratarse de allanamientos para buscar sospechosos la violencia policial no se justifica. Existió una mayoría de respuestas que consideran que la agresión física y la psicológica son tan negativas la una como la otra, porque igualmente producen daño a la persona.

Segundo presupuesto, plantea que la violencia tiene un carácter histórico y no puede entenderse fuera del contexto social que la produce. Este presupuesto implica entender de manera diferente un acto de toma por la fuerza de un edificio público en demanda de reivindicaciones sociales, del ataque a los huelguistas con bombas y armas de fuego. En este sentido Martín-Baró expresa: "Poner en el mismo saco, conceptual y valorativo, unos hechos y otros es un mecanismo ideológico que ignora el enraizamiento y naturaleza histórica de los actos de violencia”. (Martín-Baró. 1995:371).

En la línea de este presupuesto cabe recordar la reacción agresiva de la policía ante las manifestaciones de los estudiantes que se pronunciaban a favor del ICE (Instituto Costarricense de Electricidad) durante el año 2000, momento en que se discutía un proyecto de Ley para privatizar la institución. En definitiva, las reacciones de la población estudiantil en contra de la policía, no podrían compararse con otras situaciones violentas como la ocurrida en una escuela de la capital, en la que un profesor fue herido en la cara por una estudiante, cuando intervino para evitar una pelea entre dos adolescentes (La Nación, 19 de marzo del 2004).

Tercer y último presupuesto, se refiere a la "espiral de la violencia". Concepto según el cual, una vez iniciada la agresión, se activa un proceso que hace crecer la violencia de forma gradual e ininterrumpida.
Respecto de estos presupuestos, en estos últimos días (junio del 2004), se presentó en nuestro país un caso ampliamente difundido por la televisión y los periódicos, el problema entre la población civil y los policías en la "ciudadela de La Carpio", suscitado por la petición de solución de una serie de acuerdos mediante los cuales la comunidad aceptaba la apertura del relleno sanitario allí a cambio de mejoras a la comunidad, promesas que finalmente no llegaron a cumplirse. El ataque de la policía hizo que pandillas juveniles del sector se unieran para agredir a los policías y adquirieran notoriedad y mayor fuerza. La espiral de la violencia se inicia con el deseo de los dirigentes comunales de que se ponga atención a sus demandas, pero con esto se integran pandillas en el proceso, interviene la policía y el caos se profundiza y varios habitantes de la comunidad y policías resultan heridos.

Esta noticia difundida por los medios de comunicación, no sólo produjo impacto en la infancia, sino que se presentó de forma sesgada. Así, en el primer informe televisado se llegó a afirmar que eran nicaragüenses quienes propiciaban estas acciones violentas. Definitivamente, no es tan fácil medir el impacto de la noticia en la población infantil, pero una maestra de I ciclo, días después de lo sucedido, comentó que sus estudiantes, en el recreo, jugaban diciendo que eran de La Carpio, y se lanzaban piedras mientras otros corrían a esconderse.

De acuerdo con los presupuestos anteriores, cabe preguntarse sobre las posibles causas de la violencia, dado que ésta es un fenómeno que obedece a múltiples causas. La clasificación de Martín-Baró (1995) evidencia que existen diferentes situaciones de carácter emocional, ambiental y sociocultural que pueden convertirse en causas de violencia.

\section{Causas inmediatas de la violencia}

Algunas situaciones pueden convertirse en causas directas de la violencia, entre ellas: 
1. La rabia o resentimiento que tiene como base la frustración de aspiraciones y objetivos concretos. Por esta razón, siempre es recomendable buscar la existencia de frustraciones en la raíz de ciertas formas de violencia.

2. Ciertos estímulos ambientales que, enmarcados en situaciones específicas provocan actos violentos, así como las situaciones generadas por una mezcla de sujetos, objetos y simbolismos sociales.

3. La posibilidad de realizar actos violentos facilita la ocurrencia de los mismos.

4. La presión grupal.

5. La violencia como proceso asumido para alcanzar un fin.

Como se indicó anteriormente, en el caso de La Carpio, la situación social de pobreza y abandono provocada por la ausencia de políticas estatales eficaces para aminorar el hacinamiento en que vive la comunidad, unida a la acción policial para reprimir la demanda de atención y cumplimiento de las promesas gubernamentales provocaron las acciones violentas.

Cabe resaltar que La Nación del sábado 12 de junio, publicó información brindada por FLACSO acerca de la pobreza en que viven los costarricenses y nicaragüenses de esa comunidad, y un esquema porcentual sobre el país de origen de los pobladores con lo cual se presenta lo sucedido como un problema del Estado costarricense y se subraya la necesidad de brindar mejores condiciones de trabajo y de vida a las personas de esa comunidad.

Tanto el director de la escuela, como el sacerdote de la comunidad consideran urgente la apertura de un colegio vocacional. Por otro lado, un sociólogo entrevistado manifestó que el problema no se resolverá con la intervención de la policía, sino con la implementación de políticas integrales para prevenir la delincuencia y sustituir de ese modo, la infructuosa persecución de los delincuentes.

La situación vivida en La Carpio está directamente relacionada con los problemas de la pobreza, la falta de metas y espacios para la juventud, y la incapacidad del sistema educativo para mantener en las aulas a las personas en edad de estudiar, ya que sólo 40 escolares pasan a la secundaria, de 140 que estaban en el sistema de la primaria, tal como lo indica el cuadro presentado a continuación. (La Nación, sábado 12 de junio del 2004).

La situación que se da en esta comunidad, además de ejemplificar que las situaciones ambientales y de abandono son "caldo de cultivo" para el surgimiento de la violencia, lleva a reflexionar sobre la amplitud de un problema, que se inserta en

Cuadro $\mathrm{N}^{\circ} 3$

La Carpio en cifras

Datos sociales y demográficos relevantes

$\begin{array}{lr}\text { Población } & \\ \text { Habitantes } & 28.000 \\ \text { Población costarricense } & 58 \% \\ \text { Número de viviendas } & 3.940 \\ \text { Número de familias } & 4.150 \\ \text { Personas por vivienda } & 7,2 \\ \text { Agua potable } & 91,9 \% \\ \text { Abastecimiento de energía } & 95,7 \% \\ \text { Recolección pública de basura } & 91,1 \% \\ \text { Viviendas con teléfono } & 11,3 \% \\ \text { Hogares con jefatura nicaragüense } & 47 \% \\ \text { Hogares mixtos (ticos y nicas) } & 11 \% \\ \text { Ticos con empleo pleno } & 30,6 \% \\ \text { Nicas con empleo pleno: } & 38 \% \\ \end{array}$

Estado de viviendas

Hacinamiento (viviendas con exceso

de personas por habitación)

Faltante de viviendas completas

Viviendas no aptas

1.590

Total déficit de vivienda

$60 \%$

Pobreza

Pobres (leve y moderado)

$32,8 \%$

Pobreza extrema

$13,6 \%$

Sin ingresos

(indigentes o sin ingresos formales)

$12,8 \%$

Total de familias en pobreza:

$59,2 \%$ 
la constitución misma de nuestra sociedad. La educación tiene mucho trabajo que hacer en este sentido, y debe esforzarse para propiciar la inserción crítica de la persona en el mundo, y abandonar la creencia de que el acto de educar se reduce a "acomodar" los seres humanos a una realidad considerada intocable, sino a la formación de seres críticos que busquen siempre las razones del por qué ocurren las cosas. Como expresa Pablo Freire: “..si soñamos con una sociedad menos injusta, menos violenta, más humana, nuestro testimonio debe ser el de quien, diciendo no a cualquier posibilidad determinada por el destino, defiende la capacidad del ser humano de evaluar, de comparar, de escoger, de decidir y, por último, de intervenir en el mundo". (Freire, 2001:119).

En definitiva, el acto educativo es el que permite al estudiantado pensar y pensarse desde su contexto, por ello la escuela no debe permanecer como una isla en el acontecer social y la comunidad educativa, más bien debe alimentar la reflexión para actuar ante las situaciones problemáticas que puedan presentarse.

Nuestras niñas y nuestros niños no deben permanecer ajenas y ajenos al problema de la violencia y para lograrlo es importante que participen en trabajos grupales mediante los cuales analicen lo que está ocurriendo y propongan salidas a las situaciones conflictivas. Asimismo, es necesario enfatizar la formación en la resolución alternativa de conflictos, de forma tal que la población estudiantil comprenda que existen medios no agresivos para resolver los problemas.

En suma, es preciso indicar que tanto la violencia como el comportamiento agresivo son conductas adquiridas socialmente y que de acuerdo con el planteamiento de Albert Bandura, también tienen causas psicológicas. A continuación se presentan y analizan algunos factores psicológicos que pueden influir en la aparición de la violencia.

\subsection{Factores psicológicos relacionados con la violencia}

Las respuestas agresivas y los modos violentos de relacionarse entre las personas son conductas adquiridas socialmente a través de las experiencias que las niñas y los niños tienen a lo largo de su vida.

La niñez se ve sometida a acontecimientos que acrecientan la predisposición a la cultura de la agresión más que a la cultura de la paz. Esto se refleja en el hecho de que los programas televisivos y los juegos de video están llenos de agresiones. En las relaciones cotidianas, los niños suelen observar las agresiones verbales que reciben o emiten las personas que conducen automóviles, o bien las que asisten a un partido de fútbol. Asimismo, la población infantil está sometida a presenciar la desigualdad, por cuestiones de género, la burla y desprestigio de las personas a causa de la nacionalidad; y a ser testigos o víctimas del trato prepotente de quienes ostentan una posición de poder como profesores, jefes, etc. Respecto de la agresión contra las mujeres, en el año 2002 se recibieron 46012 denuncias de violencia doméstica (Proyecto Estado de la Nación, 2002).

Todas esas manifestaciones de violencia, sin lugar a dudas, demuestran que la sociedad costarricense no está conviviendo en tolerancia ni brindando a sus niños y niñas un ambiente de convivencia sano. Es evidente que la costumbre lleva a considerar como algo normal el maltrato y el irrespeto, de hecho, se tiene la creencia de que únicamente se debe intervenir cuando las acciones llevan a los golpes, pero a permanecer indiferente ante la burla, el desprecio y otras formas de agresión.

El contexto de violencia anteriormente descrito puede propiciar que el niño y la niña, a su vez, actúen de manera agresiva. $\mathrm{Al}$ respecto, cabe destacar que las investigaciones han demostrado que en el caso de los jóvenes delincuentes ha existido un modelamiento agresivo familiar. También se ha demostrado que, a través de varias 
generaciones, existe semejanza en la práctica de abuso para con las niñas y los niños.

De igual modo, los medios de comunicación masiva tienen efecto en el comportamiento agresivo:

"La tercera fuente de conducta agresiva radica en el modelamiento simbólico que proporcionan los medios de comunicación masiva, especialmente la televisión, tanto por su indiscutible predominio como por lo vívidamente que retrata los acontecimientos. El advenimiento de la televisión expandió grandemente la clase de modelos al alcance de los niños en desarrollo. Mientras sus progenitores, especialmente los de los hogares de la clase media, tenían oportunidades limitadas de observar agresiones brutales, los niños modernos han presenciado innumerables apuñalamientos, golpizas, agresiones a puntapiés, estrangulamientos, asaltos y formas menos gráficas pero igualmente destructivas de crueldad, antes de alcanzar la edad para asistir al kinder." (Bandura, 1977, 315).

El hecho de presenciar actos violentos y brutales no necesariamente harán que las niñas y los niños sigan esos patrones de conducta, pero sí puede llevar a que se conciba esta forma de trato como algo normal y por lo tanto, se asuma como aceptable dentro de las relaciones humanas.

En una entrevista abierta realizada a maestras y maestros de preescolar y primaria, éstos señalaron que los estudiantes están acostumbrados a tratarse de manera agresiva, tanto física, verbal y psicológicamente. Al respecto, destaca la opinión de una maestra que considera que dentro del mismo personal docente se dan actitudes de irrespeto hacia las alumnas y los alumnos, por ejemplo burlarse y tratarlas y tratarlos en forma sarcástica. Asimismo, expresaron que las niñas y los niños más agresivos son los que, en sus hogares, tienen algún problema de agresión o de abandono.

Las maestras y maestros consultados fueron claros al declarar su preocupación por la amplia gama de situaciones agresivas que se dan en los centros escolares. Además, expresaron que los estudiantes y las estudiantes casi no demuestran cariño y se agreden continuamente de manera verbal. Asimismo, explican que los niños y las niñas gritan y contestan de mala manera a sus maestras cuando se enojan y expusieron el caso de unos niños de quinto y sexto grado que golpearon e insultaron a un niño de preescolar, que suele molestarlos. Manifestaron, también, que si algo malo le sucede a otro niño o a otra niña, no lo consideran de su incumbencia. Un maestro de segundo grado comentó que la madre del alumno más agresivo de su clase le contó que ella y su hijo deben escaparse de la casa cuando el marido quiere golpearlos.

Ante esta realidad, es indudable que para corregir el problema se requiere un trabajo interdisciplinario, en el cual el psicólogo apoye al maestro y a la maestra para lograr una intervención adecuada en cada caso.

$\mathrm{Al}$ respecto, cabe mencionar que en la ciudadela de La Carpio, los entrevistados manifestaron opiniones acordes con lo anterior, así el sacerdote de la comunidad señaló que los altercados registrados ese día lo que hicieron fue otorgar a las pandillas un protagonismo que, en realidad no tienen, ya que no fue sino hasta ese día que se unieron para enfrentar a la policía y adquirieron mayor fuerza. Esta opinión fue compartida por el jefe policial quien manifestó que hasta ese momento, la policía había actuado cautelosamente para evitar convertirse en el enemigo de una posible coalición de pandillas.

Sin lugar a dudas, el impacto modélico de estas acciones en la población estudiantil se refleja en las apreciaciones de una maestra que encontró a alumnos de primer ciclo jugando en el recreo a "La Carpio", por lo que se lanzaban piedras y se agredían verbalmente.

Bandura (1977), en su análisis del aprendizaje social de la agresión, indica como origen de la agresión, el aprendizaje por observación, la ejecución reforzada, y las determinantes estructurales. Así, el aprendizaje de la agresión, en gran medida, se da por la observación y se consolida con el reforzamiento recibido. En otras palabras las niñas y los niños adquieren 
las conductas a través del ejemplo, o por observación indirecta, por tanto, es posible que la adquisición de conductas agresivas obedezca al hecho de haber escuchado, en algún momento, descripciones de actos violentos; no obstante, para que esto suceda, es preciso que en el ambiente social existan condiciones propicias para el desarrollo de tales conductas. Así la agresión además de ser modelada por la familia, también es reforzada por ella, de hecho, aún cuando no exista un trato agresivo hacia los hijos y las hijas, puede que el discurso dominante para resolver problemas justifique el proceder de manera violenta. Igualmente, hay ambientes donde el hecho de comportarse de manera agresiva genera la admiración de las personas hacia el niño o la niña que actúa agresivamente, lo cual no hace más que reforzar el comportamiento agresivo. Lo anterior podría ajustarse al caso de los niños que integran las pandillas de la ciudadela La Carpio. Es preciso señalar que, en ocasiones, se refuerza la conducta agresiva como una forma legítima de defenderse ante la acción violenta de otra persona. Asimismo existe otra forma de reforzar la agresividad, que consiste en presenciar el maltrato de quienes luchan por algo que creen justo. Esto puede ocurrir cuando las niñas y los niños forman grupos con la idea de defender sus derechos; otra de las posibles causas del agrupamiento en pandillas, puede ser la necesidad de unirse a otros para defenderlos por haber sido atacados injustamente. Además de lo anterior, es posible que la violencia se adquiera a través del entrenamiento que reciben los policías y militares.

Los instigadores del comportamiento agresivo se aprenden socialmente, por ello es común que ciertos grupos aprendan a odiar, discriminar y atacar a un determinado tipo de personas. Por otro lado, el momento elegido para agredir tendrá que ver con el contexto en el cual el agresor se sienta más seguro de no recibir castigo. El siguiente cuadro ilustra los conceptos antes mencionados. (Bandura 1977).

\section{Esquema $\mathrm{N}^{\circ} 1$}

Análisis del aprendizaje social de la agresión

\begin{tabular}{lll}
\hline Origen de la agresión & Instigadores de la agresión & Reforzadores de la agresión \\
\hline Aprendizaje por observación & Influencias del modelamiento & Reforzamiento externo \\
Ejecución reforzada & Desinhibitoria & Recompensas tangibles \\
Determinantes estructurales & Facilitadora & Recompensas sociales y de status \\
& Activadora & Expresión de las heridas \\
& Acrecentamiento del estímulo & Alivio de tratamiento aversivo \\
& Tratamiento aversivo & Reforzamiento vicario \\
& Asaltos físicos & Recompensa observada \\
& Amenazas e insultos & Castigo observado \\
& Reducción adversa del & Autorreforzamiento \\
& Reforzamiento & \\
& Frustración & Autocastigo \\
& Móviles de incentivo & Autorrecompensa \\
& Control por instrucciones & Neutralización del autocastigo \\
& Control simbólico y grotesco & Comparificación moral \\
& & Difusión de la responsa rabilidad \\
& Deshumanización de la víctima \\
& Atribución de culpa a las víctimas \\
& Mal interpretación de las
\end{tabular}

Diagrama que describe los orígenes, los instigadores y los reforzadores de la agresión dentro de la teoría del aprendizaje social.

Tomado de Bandura, 1977:311. 
El trabajo interdisciplinario para abordar los problemas de violencia en las escuelas es indispensable para lograr un ambiente deshinibitorio de las conductas agresivas, tarea que, de todas formas, no es fácil de llevar a cabo debido a todos los reforzadores sociales que existen a favor de la violencia, de ahí la importancia de que el cuerpo docente reciba apoyo de personal calificado en la atención a estudiantes que presentan conductas muy agresivas. A la luz de lo anterior el trabajo en la resolución alternativa de conflictos es una de las estrategias más necesarias para lograr que los estudiantes y las estudiantes, progresivamente, adquieran formas alternativas de resolver sus diferencias.

La preocupación por implementar mecanismos que aminoren las conductas agresivas en los establecimientos docentes ha llevado a proponer medidas que pueden servir como punto de partida para idear estrategias que ayuden a tratar el problema en el propio contexto. En este sentido, no se debe olvidar que la solución al problema de fondo es trabajar en pro de una mayor justicia social.

En el siguiente apartado, se presentan algunas estrategias derivadas de estudios realizados en Estados Unidos, en España y en nuestro país, tendientes a luchar contra el problema de la violencia en los centros educativos.

\section{Lineamientos para enfrentar el problema de violencia en los centros escolares}

Los lineamientos para enfrentar la violencia pueden extraerse de las conversaciones con maestras y maestros quienes han tenido que buscar formas para ayudar, de alguna manera, a la población infantil que se encuentra inmersa en situaciones de violencia. También es importante señalar que el Ministerio de Educación Pública ha preparado un nuevo material para apoyar al profesorado; dicho material es distribuido una vez que el personal docente recibe la capacitación sobre el tema.

Dado que el problema de la violencia es de carácter internacional, existe una gran cantidad de material referido al tema, por ejemplo, las investigaciones realizadas en la Universidad de Nueva York acerca de las estrategias para enfrentar la violencia en los centros educativos y las propuestas de la Universidad de Sevilla, España, referidas a los programas preventivos y de atención a las víctimas de la violencia.

De las dos propuestas se han extraído los elementos que pueden ofrecer un aporte para nuestra población docente, en el entendido de que cualquier sugerencia debe interpretarse en una situación y contexto particular.

\subsection{Estrategias a seguir en los centros educativos}

En las universidades de los Estados Unidos se han realizado investigaciones sobre el tema de la violencia en los centros escolares; Karen Osterman (2003), investigadora de la Universidad en Nueva York, señala que las situaciones de agresión y de violencia deben ser atendidas con prontitud, pero sobre todo, que es posible prevenir estas reacciones cambiando la forma en que se relaciona el personal docente con el estudiantado.

Entre otras cosas, esta investigadora constató que existen estudiantes con baja habilidad para interactuar con sus compañeras y compañeros, y que además de que no tienen relación con compañeros durante la jornada escolar, realmente no cuentan con muchos espacios para facilitar estos contactos. También se percató de que el profesorado no demuestra gran preocupación por este hecho.

La investigadora analizó más de 150 investigaciones referidas al tema de la violencia y obtuvo puntos muy importantes para tomar decisiones respecto de cómo prevenir la violencia en los centros educativos. 
Algunos de estos puntos son:

- La buena relación del educador con la estudiante o el estudiante provoca un efecto positivo en el aprendizaje; además la relación entre compañeros y compañeras tiene un efecto significativo en la salud emocional, en tanto que el rechazo tiene un efecto altamente negativo.

- Los estudiantes de escuela elemental y secundaria no tienen amigos ni amigas y no pertenecen a ningún grupo dentro de la institución educativa.

- Los estudiantes que sufren rechazo de sus compañeros o compañeras también son rechazados por sus profesores y profesoras, y otros adultos en la escuela. Estas personas llegan a estar cada vez más aisladas, ya que incluso evitan participar en eventos extra curriculares.

- Un punto extremadamente importante es que si se ofrece oportunidad a la gente para que interactúe, llega a conocerse y a ser más tolerante, aunque no llegue a tener amistad. A continuación, se señalan diversas estrategias a seguir en los centros educativos:

- Establecer claramente las reglas del grupo, de forma tal que comportamientos como la victimización, el acoso, el abuso y la agresión no sean aceptables, en ningún momento, en la institución. Evitar la creencia -bastante extendida- de que las conductas agresivas son parte del desarrollo normal de la niñez. O bien, que la criatura agredida es responsable del acto violento; a su vez, es necesario procurar que las conductas violentas no sean pasadas por alto.

- Promover una cultura de tolerancia y aceptación, y prevenir el abuso hasta en lo más mínimo. Ir más allá de la tolerancia para promover el respeto y el cuidado, a través de las palabras, pero también a través de las acciones. Enfatizar el modelamiento correcto y el reconocimiento más que el control y el castigo.

- Reforzar la seguridad de las personas que son diferentes, ya que éstas necesitan sentir que pertenecen a un lugar y que la gente se preocupa por ellas. Asimismo, el estudiantado necesita sentir que es competente. Esto, por cuanto muchas investigaciones han demostrado que en las clases se dan pocas ocasiones para que las personas menos dotadas tengan oportunidad de ser involucradas positivamente con sus compañeros, compañeras, y con el personal docente, y de este modo, sentirse bien con ellas mismas. Al involucrar más a este tipo de estudiantes con el resto de la clase y propiciar una mejor relación con los profesores y las profesoras se logra un mejor desempeño y aprovechamiento de sus talentos; lo cual, sin lugar a dudas, ayuda a que sus compañeros y compañeras los valoren cada vez más. Así, quienes inicialmente eran rechazados o rechazadas e ignorados o ignoradas por el resto de la clase, llegan a sentir un mayor respeto y consideración por parte de sus compañeros y compañeras.

- El personal docente juega un papel primordial en el desenvolvimiento de las estudiantes y los estudiantes que son rechazados o ignorados en la clase. Definitivamente, la relación que tenga el profesorado con estas personas afectará el trato que les dé el resto de los compañeros y compañeras.

- Como respuesta natural al rechazo, la población estudiantil procede de manera negligente. Este 
comportamiento es absolutamente comprensible, pues si bien los adultos se deprimen cuando sus colegas no los aprecian, el rechazo para los jóvenes puede ser mucho más significativo ya que su universo social gira en torno a la escuela y las relaciones que mantienen en dicho espacio.

- Proveer oportunidades para que el alumnado llegue a conocerse, ya que existen estudiantes que son aislados y en las clases no tienen oportunidad de intercambiar opiniones y sentimientos con otros compañeros y otras compañeras. Dicho intercambio debe realizarse a través de estrategias que involucren al estudiantado, a la comunidad escolar, profesores, bibliotecarios, secretarias, personal administrativo, etc.

En suma, es necesario hacer de las escuelas lugares donde la niñez se sienta emocionalmente segura.

En la entrevista realizada, las maestras y los maestros expresaron la importancia de mantener comunicación con el hogar de las estudiantes y los estudiantes a fin de darles seguimiento académico y emocional. Nuevamente se destaca lo expresado acerca del trato que dan algunas docentes y algunos profesores a sus alumnos y alumnas, ya que no promueven el respeto por los sentimientos del niño o la niña y suelen descalificarlos o descalificarlas frente al grupo de compañeros y compañeras.

A través de la experiencia adquirida en la educación primaria, puede señalarse que una estrategia útil para propiciar la inserción normal de los niños y las niñas que muestran comportamientos agresivos, consiste en involucrarles como ayudantes para las actividades rutinarias de la clase. Otra estrategia consiste en dedicar un rato en los recreos y tiempos libres para conversar acerca de cualquier tema, de forma tal que sus compañeros y compañeras vean que se les está dando atención cuando no proceden de manera agresiva. Por lo general, esto hace que otros niños y niñas se involucren en la conversación y se modelen, así, comportamientos adecuados de interrelación.

Otra estrategia que surte buenos resultados en la integración de los alumnos y las alumnas, consiste en integrar a los niños y las niñas rechazados y rechazadas mediante el descubrimiento de alguna habilidad especial, ya sea en el deporte o en las artes y la realización de eventos donde puedan demostrar su talento en esas áreas.

El problema de atención a la violencia escolar es muy serio, preocupante y difícil de solucionar debido a que son muchas las variables que intervienen en cada situación que se presenta; por lo tanto, se hace necesario ofrecer sugerencias para plantear, en el nivel institucional, programas preventivos y de atención a víctimas.

\subsection{Programas preventivos y de atención a víctimas y agresores}

En España se implementó un proyecto para prevenir el maltrato y la agresión entre el estudiantado. La Universidad de Sevilla ha puesto en práctica el proyecto Sevilla Anti-Violencia Escolar (SAVE), en diez escuelas que fueron parte de las veintitrés instituciones donde se realizó la investigación previa al proyecto.

Dicho proyecto busca mejorar las relaciones interpersonales y prevenir los problemas de maltrato entre escolares mediante la implementación de cuatro programas, tres de ellos preventivos y uno de atención a estudiantes víctimas de agresión y a estudiantes que actúan de manera agresiva. Los programas preventivos mencionan los ejes de fortalecimiento de la convivencia asertiva: educar sentimientos, actitudes y valores, propiciar la actividad de grupo cooperativo y desarrollar la gestión democrática de la convivencia. En el programa de atención a víctimas y agresores se hace 
hincapié en la participación de orientadores y psicopedagogos en la atención directa de los niños y niñas agresores o víctimas de agresión, y en la consejería y asesoramiento de los docentes, ayudándoles a dar seguimiento a quienes presentan conductas agresivas.

A continuación se presentan los ejes de fortalecimiento de convivencia asertiva de los programas preventivos

- Gestión democrática de la convivencia, es decir, apertura de espacios para la negociación de normas y resolución de conflictos de manera alternativa.

- Trabajo en grupo corporativo, estrategia cuyo objetivo es promover el aprendizaje por medio de la interacción y cooperación y no por medio de la competencia individual.

- Educación de sentimientos y valores tendiente a desarrollar contenidos referidos a las emociones, los sentimientos, las actitudes, los valores y en el conocimiento de sí mismo.

El programa de intervención directa con escolares en riesgo de sufrir agresiones está a cargo de psicólogas, psicólogos, orientadores y orientadoras y consiste en utilizar métodos de mediación, programas de habilidades sociales, programas de juego de roles y resolución alternativa de conflictos.

La implementación del proyecto no exige que todos los programas se den a la vez, de hecho, es factible ponerlos en práctica en la medida que el personal docente se sienta preparado para asumir cada programa.

Cabe destacar que el proyecto cuenta con la participación de toda la comunidad educativa, incluyendo padres y madres de familia. Además, las acciones preventivas del alumnado se fortalecen mediante los tres grandes ejes de la convivencia asertiva. La atención a la población estudiantil que está en riesgo social-situación detectada a través del diagnóstico- recibe un trato adecuado a sus necesidades.

Esos tres procesos preventivos y de atención a víctimas de violencia, se dan integrados dentro del currículo habitual, por lo que los grupos de docentes trabajan en equipo para insertar adecuadamente estos programas dentro de las actividades curriculares (Ortega, R. 1977) Este aspecto se ilustra a través del siguiente esquema:

Esquema $\mathrm{N}^{\circ} 2$

Esquema general del proyecto Sevilla Anti-Violencia Escolar (SAVE)

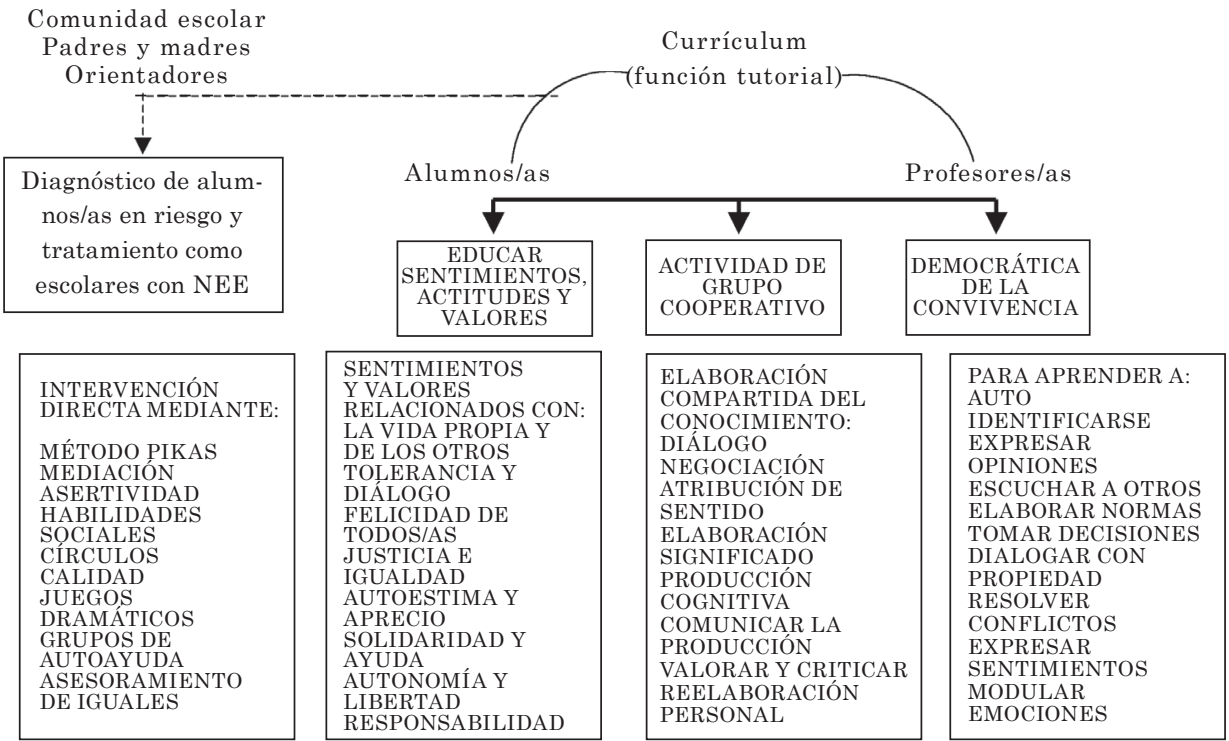

Tomado de la Revista de Educación, 313 (1997). Instituto Nacional de Calidad y Evaluación. Madrid. 
En lo que se refiere a los programas preventivos, el proyecto se acerca bastante a la propuesta del gran eje transversal de los valores y los temas transversales del currículo, propuesto en los programas de estudio de nuestro país. No obstante, aún es preciso determinar a fondo cuáles estrategias son las más adecuadas para atender a los estudiantes y las estudiantes que presentan conductas agresivas y se convierten en la pesadilla del profesorado y de sus compañeras y compañeros, ya que, en definitiva, el recrudecimiento de las medidas disciplinarias no es la alternativa más apropiada.

Al respecto, las estrategias sugeridas por la investigadora estadounidense Osterman, referidas a la preocupación por dar a cada estudiante un lugar importante dentro del plantel escolar, resultan de sumo interés en la medida de que buscan que el estudiantado encuentre un sitio importante en el cual se sienta respetado y querido.

En nuestro país, el Programa de prevención y atención de la violencia, creado en el año 2000, ha tenido el propósito de lograr una mayor cobertura en el nivel nacional.

En el marco de dicho programa, las escuelas de nuestro país han trabajado, básicamente, con material impreso consistente en tres libros:

- La familia: componente vital en la prevención de la violencia intrafamiliar. Este libro es una guía para que los docentes y las docentes trabajen con las familias utilizando una metodología participativa. El contenido de la guía se desarrolla en 12 sesiones de trabajo con los padres y madres de familia.

- Si nos levantan la mano que sea para abrazarnos. Guía para el personal docente de I y II ciclos. Este libro presenta sugerencias en el tratamiento de los contenidos temáticos de las materias básicas, específicamente, reflexiones acerca de situaciones que promuevan el reconocimiento de situaciones de violencia y alternativas para enfrentar estas situaciones en la casa y en la escuela, asimismo, busca crear conciencia en el alumnado acerca de su derecho a vivir libre de violencia.

- Guía para la prevención de la violencia desde el contexto educativo. Este libro consta de cinco módulos creados para ser utilizados en talleres infantiles, de modo que los niños y las niñas se conviertan en promotores de la no violencia en su contexto escolar. La propuesta es realizarlos, todos, en una semana o bien, un taller por semana, en horario extraclase.

Durante el año 2004, además de lograr una mayor cobertura con el Programa, el personal docente que ha participado en la capacitación respectiva, ha recibido un nuevo material, que incluye tres módulos creados por el Ministerio de Educación en conjunto con la Fundación Paniamor. Los módulos contienen el marco teórico y jurídico, y los objetivos y modelos de la educación para la paz.

La capacitación para utilizar este nuevo material ha sido sistemática en la Regional de Cartago, donde 25 escuelas distribuidas en los diferentes circuitos correspondientes a la regional, han recibido la capacitación.

La implementación de este Programa es responsabilidad del docente o la docente de cada nivel, pero la comisión encargada de supervisar y retroalimentar su labor está formada por los maestros y las maestras que deseen integrarla, y la orientadora o el orientador, el director o la directora de la institución y, si existe, la profesional especializada en problemas emocionales. En la dirección regional el Programa está a cargo de la asesoría de Orientación.

En definitiva, existen caminos para implementar eficazmente una educación para la paz y aminorar los niveles de violencia; sin embargo, lo más importante es que dicha labor involucre a toda la sociedad. 


\section{Conclusiones y recomendaciones}

Existen causas sociales de la violencia relacionadas con problemas sociales de pobreza y las instancias asociadas a esta condición, por consiguiente, es importante la acción gubernamental en este sentido, así como el fortalecimiento de los programas de ayuda a la población estudiantil, entre ellos los comedores escolares y el transporte hacia los centros educativos. Asimismo, es preciso fortalecer y evaluar las alternativas educativas para la población que ha desertado del sistema escolar, por ejemplo el Programa Nuevas Oportunidades y Aula Abierta. Si bien existen muchos textos e investigaciones respecto a la importancia de revisar los programas agresivos que ofrece la televisión y los efectos que produce en las mentes infantiles, es importante tener en cuenta que el manejo de la información por parte de los medios de comunicación masiva implica un impacto importante en la población, por tanto, sería importante tomar medidas concretas en este aspecto. El documental del cineasta Michael Moore "Masacre en Columbine" que denuncia la violencia en los centros escolares de los Estados Unidos, demuestra cómo los medios informativos influyen mucho en la actitud de la ciudadanía respecto de su comportamiento defensivo o agresivo frente a los demás.

La actitud del grupo de docentes es fundamental para enfrentar el problema de la violencia, ya que las guías elaboradas por el Ministerio de Educación Pública necesitan que las maestras y los maestros se involucren con entusiasmo en ese trabajo. De igual modo, es preciso buscar la asesoría y apoyo de expertos y expertas o textos referidos al tema. El medio electrónico Internet es un espacio donde se puede conseguir fácilmente información de apoyo para atender las necesidades que se presentan, sobre todo, el manejo alternativo de conflictos, base para la intervención en caso de que surjan problemas entre estudiantes.
En cuanto a las causas psicológicas de la violencia, es importante propiciar el abordaje grupal de las problemáticas escolares. Así, en el caso de estudiantes que son rechazados, el trabajo en equipo de todo el cuerpo docente que habitualmente se relaciona con ellos, fortalecerá la implementación de estrategias para lograr que esta población estudiantil se sienta apoyada y más segura desde el punto de vista emocional. En ocasiones sucede que una niña o un niño está pasando por una crisis familiar y el maestro o la maestra encargada no informa al resto del grupo de docentes que trabajan con el estudiante o la estudiante. Esta falta de comunicación hace que el afectado o la afectada no reciba el apoyo necesario en el momento que lo necesita por parte de todos los involucrados en la institución escolar.

Cualquier medida que pueda implementarse en los centros escolares para apoyar el desarrollo de la fortaleza emocional de la población estudiantil, es importante, de hecho dichas estrategias tendrán mejor resultado si se involucra a toda la comunidad educativa y la familia. En este sentido cabe resaltar que el trabajo en equipo facilita también el descubrimiento de destrezas especiales en los estudiantes y las estudiantes, las cuales, a su vez permiten fortalecer el autoestima y las destrezas sociales en la relación con los compañeros y las compañeras, aspectos fundamentales para el sano desarrollo emocional de los niños y las niñas.

Es preciso considerar que además de la preocupación que genera el tema de la violencia, existe material para enfrentar el problema en los centros escolares y que mediante la participación de la comunidad, los padres, madres y docentes comprometidos, es posible implementar en nuestro país una cultura de paz que tal como expresa Johan Galtung, no significa la desaparición de los problemas o los conflictos, pero sí el saber negociar para no recurrir a la violencia al momento de resolverlos. En su discurso pronunciado en Argentina Galtung describió la paz positiva como aquélla que: 
"presupone un nivel reducido de violencia directa y un elevado nivel de justicia. Se busca armonía social, justicia e igualdad y la eliminación de la violencia estructural, concepto que alude a la violencia generada por las estructuras, es decir, por la desigualdad existente dentro de la sociedad que impide a las personas satisfacer sus necesidades fundamentales espirituales y materiales." (Galtung, 2001).

Sin lugar a dudas, el tema de la violencia en las escuelas requiere continuar con la capacitación de los docentes y las docentes y el resto de la comunidad en la resolución alternativa de conflictos, de forma que ésta se vuelva parte de la cultura dentro de las instituciones educativas y fuera de ellas. Asimismo, la escuela debe recordar que su norte es la formación de personas críticas que puedan expresarse libremente y comprometerse con su palabra para lograr una sociedad más justa y respetuosa de los derechos humanos, y la justicia social. En suma, la idea es formar personas asertivas en cuanto a la forma de relacionarse con otras personas y con el medio ambiente, personas que estén atentas a descubrir y denunciar la violencia encubierta que produce daños profundos a toda la sociedad.

\section{Referencias bibliográficas}

Bandura, Albert. Modificación de conducta. México: Trillas. 1977.

Freire, Paulo. Pedagogía de la indignación. Madrid: Morata. 2001.

Brenes, Raymudo y Grillo, Milena. Fundación Paniamor. Manual de contenidos: violencia y abuso contra personas menores de edad. San José: C. R. Proniño. 1998.

Larousse. Diccionario El Pequeño Larousse. Colombia: Printer Colombiana S.A. 2003.

Galtung, Johan. Conferencia. "El conflicto en la era de la globalización”. 2001 Recuperado el 4 de julio del 2004, de http//www.respuestaparalapaz.org. ar/conferencia.htm

Herrera, M. "La Carpio, ni cueva, ni gueto, ni amenaza". La Nación, pp. 19 A 2004, 12 de julio.

Martín-Baró, Ignacio. Acción e ideología. Psicología social desde Centroamérica. San Salvador: UCA Editores. 1995.

Ortega, R. "El proyecto Sevilla anti-violencia escolar". Revista de Educación. Instituto Nacional de Calidad $y$ Evaluación. Nº 313. Madrid. 1997.

Osterman, K. "Less isolation, less violent students". Education Digest, 68 (9) pp. 18. 2003. Recuperado el 8 de abril 2004 desde EBSCO host database (Academic Search Premiere).

Proyecto Estado de la Nación. Noveno informe sobre el estado de la Nación en desarrollo humano sostenible, año 2002. San José: C. R. Proyecto Estado de La Nación. 2003.

Vargas, O. "La Carpio, ni cueva, ni gueto, ni amenaza”. La Nación, pp. 14ª 2004,19 de marzo. 
